\title{
UCRL-JRNL-204073
}

LAW RENCE LIVERMORE N A T IO N A L LABORATORY

\section{A Study of Microstructural Length Scale Effects on the Behaviour of FCC Polycrystals Using Strain Gradient Concepts}

K. S. Cheong, E. P. Busso, A. Arsenlis

May 10, 2004

International Journal of Plasticity 
This document was prepared as an account of work sponsored by an agency of the United States Government. Neither the United States Government nor the University of California nor any of their employees, makes any warranty, express or implied, or assumes any legal liability or responsibility for the accuracy, completeness, or usefulness of any information, apparatus, product, or process disclosed, or represents that its use would not infringe privately owned rights. Reference herein to any specific commercial product, process, or service by trade name, trademark, manufacturer, or otherwise, does not necessarily constitute or imply its endorsement, recommendation, or favoring by the United States Government or the University of California. The views and opinions of authors expressed herein do not necessarily state or reflect those of the United States Government or the University of California, and shall not be used for advertising or product endorsement purposes. 


\title{
A Study of Microstructural Length Scale Effects on the Behaviour of FCC Polycrystals Using Strain Gradient Concepts
}

\author{
K. S. Cheong ${ }^{a, 1}$, E. P. Busso ${ }^{a, *}$, A. Arsenlis ${ }^{b}$ \\ ${ }^{a}$ Department of Mechanical Engineering, Imperial College London, SW7-2BX, UK \\ ${ }^{\mathrm{b}}$ Lawrence Livermore National Laboratory, University of California, Livermore, \\ $C A, U S A$
}

\begin{abstract}
Grain size is a critically important aspect of polycrystalline materials and experimental observations on $\mathrm{Cu}$ and $\mathrm{Al}$ polycrystals have shown that a Hall-Petchtype phenomenon does exist at the onset of plastic deformation. In this work, a parametric study is conducted to investigate the effect of microstructural and deformation-related length scales on the behaviour of such FCC polycrystals. It relies on a recently proposed non-local dislocation-mechanics based crystallographic theory to describe the evolution of dislocation mean spacings within each grain, and on finite element techniques to incorporate explicitly grain interaction effects. Polycrystals are modeled as representative volume elements (RVEs) containing up to 64 randomly oriented grains. Predictions obtained from RVEs of $\mathrm{Cu}$ polycrystals with different grain sizes are shown to be consistent with experimental data. Furthermore, mesh sensitivity studies revealed that, when there is a predominance of geometrically necessary dislocations (GNDs) relative to statistically-stored dislocations (SSDs), the polycrystal response becomes increasingly mesh sensitive. This was found to occur specially during the early stages of deformation in polycrystals with small grains.
\end{abstract}

Key words: Crystal Plasticity, Dislocations, Constitutive Behaviour, Polycrystalline Material

\footnotetext{
* Corresponding author. Tel: +44-207-594-7084; Fax: +44-207-594-7017

Email address: e.busso@imperial.ac.uk (E. P. Busso).

1 Present address : Industrial Research Ltd., Lower Hutt 6009, New Zealand.
}

Preprint submitted to Int. Journal of Plasticity

Dec 2003 


\section{Introduction}

In polycrystalline metal aggregates, grain-size strengthening - the so-called Hall-Petch effect - is commonly observed (Hall, 1951; Petch, 1953). Experimental evidence of such size-dependent effects (e.g. Fleck et al., 1994), have shown that the material response is controlled by deformation gradients. When the gradients are of the order of the dominant geometric or microstructural length scale, such as the average grain size in a polycrystal, the overall stress scales with decreasing grain size for a given strain. In the case of metallic polycrystals, strain gradients arise primarily due to the lattice incompatibilities associated with the inhomogeneous plastic deformation between neighboring grains. In order to accommodate these strain gradients, generation of geometrically necessary dislocations (GNDs) is required in these regions of incompatibility (Ashby, 1970). The introduction of these GNDs, in addition to those statistically-stored dislocations (SSDs), which are inherently random in nature, results in additional strengthening of the material. Such gradientdependent behaviour is expected to become important when the deformation gradients become sufficiently large with respect to the controlling microstructural feature. Hence, polycrystals with a finer grain size develop strain gradients which extend further into the grain, exhibiting a stronger response due to the additional presence of GNDs associated with such gradients.

Strain gradient plasticity concepts are commonly used to study length scale effects in polycrystalline metallic aggregates and a number of non-local continuum mechanics theories have been formulated to address these effects. Phenomenological theories incorporating higher-order strain gradients, couple stresses as well as requiring higher-order boundary conditions, were put forward by Fleck et al. (1994), Gurtin (2003) and Gudmundson (2004), to predict the strain gradient dependence of strength. An alternative and more physically intuitive approach to describe strain gradient effects without the need to include higher order stresses or additional boundary conditions was developed by several authors (e.g. Dai and Parks, 1997; Busso et al., 2000; Bassani, 2001; Arsenlis and Parks , 2001; Acharya and Beaudoin, 2000; Huang et $a l ., 2004)$. Here, strain gradient effects are introduced directly into the evolutionary laws of the internal slip system state variables. This type of strain gradient theory has been shown to be capable of providing great physical insight into the effects of microstructure on the observed macroscopic phenomena, including rate-independent plastic deformation and visco-plasticity in both single crystal and polycrystalline materials (e.g. Busso and Cheong, 2001; Meissonnier et al., 2001). They are relatively easy to implement numerically and do not require higher order stresses or additional boundary conditions. However, they are unable to describe problems which may require non-standard boundary conditions, such as the boundary layer problem modelled by Shu et al. (2001). Furthermore, although they have been successful in

explaining length scale dependence phenomena, the link between the differ- 
ent microstructural and deformation-related length scales, and finite-element mesh sensitivity has never been systematically addressed. For instance, in the grain-size effect study by Acharya and Beaudoin (2000) on FCC and BCC polycrystals, only the mesh-insensitive polycrystal response for one particular grain size was shown. Similarly, mesh sensitivity studies on two-phase superalloy single crystals by Meissonnier et al. (2001) were only carried out for one precipitate size.

In this work, a parametric study is conducted to investigate the effect of microstructural and deformation-related length scales on the behaviour of $\mathrm{Cu}$ polycrystals, including a systematic investigation of the mesh sensitivity of the finite element (FE) results over a spectrum of length scales. The nonlocal continuum rate-dependent theory presented here follows from the work

of Busso et al. (2000), where the evolution of the GNDs is linked to local slip rate gradients and incorporated into the evolutionary behaviour of the slip resistance, one of the internal state variables of the crystallographic model. In addition, a novel dislocation-mechanics based crystallographic theory will be used to describe the evolution of mean spacings between dislocations in each grain and the average behaviour of the aggregates' representative volume elements.

\section{Gradient and rate-dependent constitutive framework}

The strain gradient and rate-dependent constitutive framework for finite strains is presented in this section. It relies on the multiplicative decomposition of the total deformation gradient referred to the undeformed crystal configuration, $\mathbf{F}$, into an inelastic component associated with pure slip, $\mathbf{F}^{\mathbf{p}}$, while the lattice remains undistorted and unrotated, and an elastic component, $\mathbf{F}^{\mathbf{e}}$, which accounts for the elastic stretching and rigid-body rotations,

$$
\mathbf{F}=\mathbf{F}^{\mathbf{e}} \mathbf{F}^{\mathbf{p}}
$$

From the kinematics of dislocation motion, the rate of change of the inelastic deformation gradient is given by,

$$
\dot{\mathbf{F}}^{\mathbf{p}}=\left(\sum_{\alpha=1}^{N} \dot{\gamma}^{\alpha} \mathbf{P}^{\alpha}\right) \mathbf{F}^{\mathbf{p}} \quad \text { with } \quad \mathbf{P}^{\alpha} \equiv \mathbf{m}^{\alpha} \otimes \mathbf{n}^{\alpha},
$$

where the sum extends over $N$ active slip systems, $\dot{\gamma}^{\alpha}$ is the slip rate in a slip system $\alpha$, while $\mathbf{m}^{\alpha}$ and $\mathbf{n}^{\alpha}$ refer to the slip direction and the slip plane normal unit vectors, respectively. The constitutive stress-strain relation under isothermal conditions is,

$$
\mathbf{T}=\mathcal{L}: \mathbf{E}^{\mathrm{e}}, \text { with } \mathbf{E}^{\mathrm{e}}=\frac{\mathbf{1}}{\mathbf{2}}\left(\mathbf{F}^{\mathrm{eT}} \mathbf{F}^{\mathbf{e}}-\mathbf{1}\right)
$$


where $\mathbf{E}^{e}$ is the Green-Lagrange tensorial elastic strain measure, $\mathcal{L}$ is the anisotropic elastic moduli, $\mathbf{1}$, the second order identity tensor, and $\mathbf{T}$ is the second Piola-Kirchoff stress tensor. The latter is related to the Cauchy stress tensor $\boldsymbol{\sigma}$ through

$$
\sigma=\operatorname{det}\left(\mathbf{F}^{\mathrm{e}}\right)^{-1} \mathbf{F}^{\mathrm{e}} \mathbf{T} \mathbf{F}^{\mathrm{e} \mathbf{T}}
$$

The formulation is completed with the flow and evolutionary equations to describe the behaviour of each individual slip system. The form of the flow rule exhibits an explicit dependence of the activation energy on a driving stress, $\tau_{t h}^{\alpha}$, which takes into account the effects of lattice friction and thermally-activated obstacles. Here,

$$
\tau_{t h}^{\alpha}=\tau^{\alpha}-S^{\alpha} \mu / \mu_{0}
$$

where $\tau^{\alpha}$ is the resolved shear stress and $S^{\alpha}$ is the total resistance to slip in the generic slip system $\alpha$. The ratio $\mu / \mu_{0}$ represents the shear moduli ratio at the absolute temperatures $\theta$ and $0 \mathrm{~K}$, respectively. The slip rate in each slip system $\alpha, \dot{\gamma}^{\alpha}$, follows the exponential function (Busso, 1990),

$$
\dot{\gamma}^{\alpha}=\dot{\gamma}_{0} \exp \left[-\frac{F_{0}}{\kappa \theta}\left\{1-\left\langle\frac{\left|\tau^{\alpha}\right|-S^{\alpha} \mu / \mu_{0}}{\hat{\tau}_{0} \mu / \mu_{0}}\right\rangle^{p}\right\}^{q}\right] \operatorname{sgn}\left(\tau^{\alpha}\right) .
$$

Here, $F_{0}$ is the Helmholtz free energy of activation required to overcome obstacles without the aid of external work, and $\kappa$ is the Boltzmann constant. Also, $\dot{\gamma}_{0}$ is a pre-exponential term and $\hat{\tau}_{0}$ is the maximum glide resistance at which dislocations can be mobilised without the aid of thermal activation. The exponents $p$ and $q$ describe the profile of the activation energy versus the resolved shear stress function, as proposed by Kocks et al. (1975). Lastly, $\operatorname{sgn}\left(\tau^{\alpha}\right)$ accounts for either positive and negative slip on the system. In Eq. 6 , the overall slip resistance, $S^{\alpha}$, incorporates contributions from both statistically stored and geometrically necessary forest dislocations. Therefore, the total dislocation density on an arbitrary slip system is defined by,

$$
\rho_{T}^{\alpha}=\rho_{S}^{\alpha}+\rho_{G}^{\alpha}
$$

where $\rho_{S}^{\alpha}$ and $\rho_{G}^{\alpha}$ refer to the SSD and GND densities, respectively. In addition, $\rho_{S}^{\alpha}$ and $\rho_{G}^{\alpha}$ are discretised into pure edge and pure screw components. Thus Eq. 7 can be expanded to,

$$
\rho_{T}^{\alpha}=\left(\rho_{S_{e}}^{\alpha}+\rho_{S_{s w}}^{\alpha}\right)+\left(\rho_{G_{s w}}^{\alpha}+\rho_{G_{e t}}^{\alpha}+\rho_{G_{e n}}^{\alpha}\right),
$$

where $\rho_{S_{e}}^{\alpha}$ and $\rho_{S_{s w}}^{\alpha}$ further denote the pure edge and screw SSD components, $\rho_{G_{s w}}^{\alpha}$ a set of screw GNDs parallel to the slip direction, $\mathbf{m}^{\alpha}$, and $\rho_{G_{e t}}^{\alpha}$ and $\rho_{G_{e n}}^{\alpha}$, edge GND components oriented parallel to the slip system normal, $\mathbf{n}^{\alpha}$, and to $\mathbf{t}^{\alpha}=\mathbf{m}^{\alpha} \times \mathbf{n}^{\alpha}$, respectively. The evolutionary behaviour of both edge and 
screw SSDs follows from the work of Cheong and Busso (2004),

$$
\begin{aligned}
\dot{\rho}_{S_{e}}^{\alpha} & =\frac{C_{e}}{b_{S}^{\alpha}}\left[K_{e} \sqrt{\left.\sum_{\beta=1}^{N} \rho_{T}^{\beta}-2 d_{e} \rho_{S_{e}}^{\alpha}\right]\left|\dot{\gamma}^{\alpha}\right|}\right. \\
\dot{\rho}_{S_{s w}}^{\alpha} & =\frac{C_{s w}}{b_{S}^{\alpha}}\left[K_{s w} \sqrt{\sum_{\beta=1}^{N} \rho_{T}^{\beta}}-\rho_{S_{s w}}^{\alpha}\left\{K_{s w} \pi d_{s w}^{2} \sqrt{\sum_{\beta=1}^{N} \rho_{T}^{\beta}}+2 d_{s w}\right\}\right]\left|\dot{\gamma}^{\alpha}\right|,
\end{aligned}
$$

where $C_{e}$ and $C_{s w}$ describe the relative contributions to the slip produced by SSDs from edge and screw types, and $b_{S}^{\alpha}$ is the magnitude of the SSDs' Burgers vector. The parameters $d_{e}$ and $d_{s w}$ are the respective critical distances for spontaneous annihilation of opposite sign edge and screw dislocations, while $K_{e}$ and $K_{s w}$ are related to the mean free path of the edge and screw dislocations, respectively. Similarly, the evolutionary law for each set of GNDs is determined from Nye's dislocation tensor in terms of the spatial gradient of the slip rate (Busso et al., 2000),

$$
b_{G}^{\alpha}\left(\dot{\rho}_{G_{s w}}^{\alpha} \mathbf{m}^{\alpha}+\dot{\rho}_{G_{e t}}^{\alpha} \mathbf{t}^{\alpha}+\dot{\rho}_{G_{e n}}^{\alpha} \mathbf{n}^{\alpha}\right)=\operatorname{curl}\left(\dot{\gamma}^{\alpha} \mathbf{n}^{\alpha} \mathbf{F}^{\mathbf{p}}\right) .
$$

The slip resistance contributions from the SSDs and GNDs can then be determined from,

$$
\begin{aligned}
& S_{S}^{\alpha}=\lambda_{S} \mu_{0} b_{S}^{\alpha} \sqrt{\sum_{\beta=1}^{N} h_{S}^{\alpha \beta} \rho_{S}^{\beta}}, \\
& S_{G}^{\alpha}=\lambda_{G} \mu_{0} b_{G}^{\alpha} \sqrt{\sum_{\beta=1}^{n_{\alpha}} h_{G}^{\alpha \beta} \rho_{G}^{\beta}},
\end{aligned}
$$

where $\mu$ is the shear modulus, $\lambda_{S}$ and $\lambda_{G}$ are statistical coefficients which account for the deviation from regular spatial arrangements of the SSD and GND populations, respectively, and $b_{S}^{\alpha}$ and $b_{G}^{\alpha}$ the corresponding Burgers vector magnitudes. In what follows, it will be assumed for simplicity that $\lambda=\lambda_{S}=\lambda_{G}$ and $b=b_{S}^{\alpha}=b_{G}^{\alpha}$. Furthermore, $h_{S}^{\alpha \beta}$ and $h_{G}^{\alpha \beta}$ are SSD and GND interaction functions, respectively, which can be generically expressed as,

$$
h_{k}^{\alpha \beta}=\omega_{k 1}+\left(1-\omega_{k 2}\right) \delta^{\alpha \beta} \quad \text { for } \quad k=S, G,
$$

where $\omega_{k 1}$ and $\omega_{k 2}$ are the interaction coefficients, and $\delta^{\alpha \beta}$ is the Kronecker delta. The corresponding total slip resistance, $S^{\alpha}$, can be obtained from,

$$
\begin{aligned}
S^{\alpha} & =\left[\left(S_{S}^{\alpha}\right)^{2}+\left(S_{G}^{\alpha}\right)^{2}\right]^{1 / 2} \\
& =\lambda \mu b \sqrt{\sum_{\beta=1}^{N}\left[h_{S}^{\alpha \beta} \rho_{S}^{\beta}+h_{G}^{\alpha \beta} \rho_{G}^{\beta}\right]}
\end{aligned}
$$


The above crystallographic formulation has been implemented numerically into a commercial FE code (ABAQUS, 2001) using a finite strain framework and an implicit time-integration procedure to update the stresses and slip resistances at each integration point. It should be noted that the non-local effects associated with the deformation gradients at each integration point are incorporated into Eq. 16 through the current value of the GND densities. At each integration point, the evolution of the GNDs is determined from Eq. 11 with the calculated slip-rate gradients outlined by Busso et al. (2000). There, the slip rate at the integration points are extrapolated to the corner nodes of the element using linear shape functions associated with an 8-noded linear isoparametric element with full $(2 \times 2 \times 2)$ integration so that the number of corner nodes and integration points coincide. Subsequently, the slip rate gradients at the corner nodes are interpolated back to the integration points from the spatial derivatives of the linear shape functions. A schematic description of the required sequence of steps is shown in Fig. 1. Here, the term $\operatorname{curl}\{\dot{\Phi}\}$ refers to the right-hand side of Eq. 11. In passing, we note that Acharya and Bassani (2000) adopts a different approach whereby the spatial derivatives of $\mathbf{F}^{\mathbf{e}-\mathbf{1}}$ are linked explicitly to the lattice incompatibility while Cermelli and Gurtin (2001) propose $\operatorname{curl}\left(\mathbf{F}^{\mathbf{p}}\right) \mathbf{F}^{\mathbf{p}} \mathbf{T}$.

\section{Calibration of the single crystal model for copper}

The single crystal constitutive model was calibrated using high symmetry copper single crystal tensile test data. The flow rule parameters (i.e. $F_{0}, \hat{\tau}_{0}, p$, $q$ and $\dot{\gamma}_{0}$ in Eq. 6) were determined first. Following the work of Kocks et al. (1975), the pre-exponential term $\dot{\gamma}_{0}$ typically varies within the range of $10^{6}$ to $10^{7} s^{-1}$, the Helmholtz free energy of activation $F_{0}$ is typically

$$
0.05 \leq \frac{F_{0}}{\mu b^{3}} \leq 2.0
$$

while the exponents $p$ and $q$ vary within the ranges,

$$
\begin{aligned}
& 0 \leq p \leq 1 \\
& 1 \leq q \leq 2
\end{aligned}
$$

For the hardening laws (Eqs. 9 and 10), the relevant parameters are the annihilation distances for the edge and screw dislocations, $d_{e}, d_{s w}$, and the constants defining the mean free paths of the respective dislocation types, namely $K_{e}$ and $K_{s w}$. These parameters are chosen to be compatible with physical observations made at the microstructural level. The selected values for $d_{e}$ and $d_{s w}$ are $1.0 \mathrm{~nm}$ and $5.0 \mathrm{~nm}$, respectively. This value of $d_{e}$ is consistent with the estimate of $1.6 \mathrm{~nm}$ reported by Essmann and Mughrabi (1979). A higher value of $5 \mathrm{~nm}$ is taken for $d_{s w}$ based on the fact that screw dislocations have the ability to cross-slip, thus increasing the likelihood of spontaneous annihilation between screw dislocations of opposite sign. 
Previous slip line work on $\mathrm{Cu}$ by Rebstock (1957) and Mader (1957) estimated the distance travelled by edge dislocations to be approximately twice that of screw types. As this is associated with the mean free path of the dislocations, $K_{s w}$ is set to be twice the value of $K_{e}$ so that, in Eq. 10, the relationship between the mean free paths of the two dislocation types agree with these physical observations. Consequently, $K_{s w}=2 K_{e}$ and a value of $K_{e}=14.1 \times 10^{-3}$ was calibrated based on the single crystal stress-strain data, while $C_{e}$ and $C_{s w}$ were set at 0.5 to keep the slip contributions from both edge and screw dislocations equal. The initial total dislocation density specified is $16000 \mathrm{~mm}^{-2}$, which is typically of the same order as that measured in pure FCC metallic single crystals (Honeycombe, 1968). The total density is equally made up of edge and screw types and assumed to be same in each of the twelve octahedral slip systems. As shown in Fig. 2, good agreement was obtained between the predicted single crystal response and the tensile test data from [100], [111] and [112] oriented Cu single crystals (Takeuchi, 1975). The calibration was carried out at a temperature of $298 \mathrm{~K}$ and at a true strain rate of $3 \times 10^{-3} s^{-1}$. The slightly higher predictions obtained for the three orientations are likely to be due to the assumption that the $\mathrm{Cu}$ crystals were initially perfectly oriented with the [100], [111] and [112] crystal axes. A summary of the material constants and model parameters at $298 K$, is shown in Table 1. Note that of all these parameters, only three were calibrated to optimise the stress-strain predictions shown in Fig. 2.

\section{Representative volume elements of $\mathrm{Cu}$ polycrystals}

In this study, two representative volume elements (RVEs) are used to represent copper polycrystals. The first consists of a polycrystal with 8 randomly oriented grains, and the other contains 64 random grains. Both RVE models have the same initial cubic geometry and each grain has the same cuboidal shape and size, containing the same number of elements. The models are constructed using standard 20-noded isoparametric 3D elements with reduced integration. The randomly assigned grain orientations for both models are shown in the stereographic triangles of Fig. 3.

The series of cubic meshes generated for the 8-grain polycrystal is shown in Fig. 4. Here, the initial grain size is denoted by D. The coarsest mesh consisted of $2 \times 2 \times 2$ elements and subsequent mesh refinement was carried out in multiples of 8 with the finest mesh having $14 \times 14 \times 14$ elements. Note that all the meshes share identical assignments of the initial grain orientations and only differ in the number of elements used to discretise the grains. A similar description applies to the 64-grain polycrystal with the coarsest form of the model made up of a $4 \times 4 \times 4$ mesh. Two sequences of mesh refinement in multiples of 64 were used to produce finer $8 \times 8 \times 8$ and 16x16x16 meshes, which are identical in geometry to those of the 8-grain polycrystal and are therefore not shown here. 
To study the influence of grain size on mesh sensitivity, a range of grain sizes $\mathrm{D}=7.5,15,30,75$ and $150 \mu \mathrm{m}$ were considered for the 8-grain polycrystal RVE, while $\mathrm{D}=15$ and $75 \mu \mathrm{m}$ were used for the 64 -grain polycrystal. Periodic boundary conditions were applied to the external faces of the cuboidal mesh to accomodate a general deformation state in the polycrystal. In all cases, each grain of the polycrystal is described by the $\mathrm{Cu}$ single crystal model calibrated in the previous section. A displacement history is applied to the $X_{3}$ face of the RVE models (see Fig. 4) so as to give a true strain rate of $1.0 \times 10^{-3} s^{-1}$. All simulations were carried out at a temperature of $298 \mathrm{~K}$.

\section{Predicted polycrystal response}

The predicted polycrystal response and the results of the mesh sensitivity study are presented in this section. Figure 5 shows the grain size effect on the polycrystal response for the 8-grain polycrystal calculated with the finest $\mathrm{FE}$ mesh (i.e. $14 \times 14 \times 14$ elements). It is observed that a reduction in the initial grain size from 150 to $7.5 \mu \mathrm{m}$ strengthens the polycrystal. This effect is small at relatively large grain sizes $(\mathrm{D}=75,150 \mu \mathrm{m})$ and increases with decreasing grain size. Figures 6(a) to (e) show the effect of mesh refinement on the polycrystal response for different initial grain sizes. For the two largest grains, namely $\mathrm{D}=75$ and $150 \mu \mathrm{m}$ (see Figs. 6(a) and (b)), the predicted responses are relatively mesh insensitive. However, when $\mathrm{D} \leq 30 \mu \mathrm{m}$, the polycrystal response increases with mesh refinement. It can also be seen from the circled regions in Figs. 6(c) to (e), that the polycrystal response becomes mesh sensitive only after a small amount of strain. In the case of $\mathrm{D}=7.5 \mu \mathrm{m}$, the predictions exhibit mesh sensitivity as soon as plastic deformation occurs.

A summary of these results can be seen in Fig. 7, where the predicted flow stress $\sigma_{33}$ for different values of $\mathrm{D}$ at $\varepsilon_{33}=0.10$ have been plotted against a measure of the extent of mesh refinement, henceforth defined by the ratio $\eta$ between $\mathrm{D}$ and the element size. It can be seen that polycrystals with $\mathrm{D}=$ 7.5 and $15 \mu \mathrm{m}$ are highly dependent on the ratio $\eta$, showing an appreciable increase in the flow stress with decreasing element size. On the other hand, the polycrystal response for $\mathrm{D}=75$ and $150 \mu \mathrm{m}$ are practically insensitive to mesh size. Figures $8(\mathrm{a})$ and (b) show that the mesh sensitivity results on the 64-grain polycrystal are similar to those on the 8-grain polycrystal when $\mathrm{D}=$ $75 \mu \mathrm{m}$ and $15 \mu \mathrm{m}$, respectively. In Fig. 8(a), the stress-strain responses with D $=75 \mu \mathrm{m}$, stabilise and approach a mesh-insensitive solution, whereas in Fig. 8(b), when $\mathrm{D}=15 \mu \mathrm{m}$, it increases with mesh refinement in the direction of the arrows. It should also be noted that the stronger predicted response of the 64-grain polycrystal is linked to the larger number of randomly-oriented grains used to represent the RVE. If the number of grains were increased further, a response independent of the number of grains would be expected. 


\section{Discussion}

\subsection{Effect of initial dislocation density}

This discussion will focus on understanding the cause(s) for the lack of mesh convergence, observed in some of the above cases. As previously discussed, the $\mathrm{Cu}$ polycrystal response is mesh insensitive when strain gradient effects are small (i.e. $\mathrm{D}=75,150 \mu \mathrm{m}$ ) but becomes mesh sensitive when $\mathrm{D}$ $\leq 30 \mu \mathrm{m}$, in particular at smaller strains. This suggests that the initial buildup of GNDs at the onset of deformation far exceeds the SSD population and consequently, controls the polycrystal flow stress. Since the initial dislocation density in a grain is assumed to consist entirely of SSDs, the effect of the initial dislocation density, $\rho_{i}^{\alpha}$, on the polycrystal response is assessed by defining it in terms of the reference value $\rho_{r e f}^{\alpha}=16,000 \mathrm{~mm}^{-2}$ from the single crystal model calibration.

Figure 9 shows the polycrystal responses using the coarsest $4 \times 4 \times 4$ and finest $14 \mathrm{x} 14 \mathrm{x} 14$ meshes when $\rho_{i}^{\alpha}=\rho_{\text {ref }}^{\alpha}$ and $\rho_{i}^{\alpha}=625 \rho_{\text {ref }}^{\alpha}$ for $\mathrm{D}=7.5,15$ and $30 \mu \mathrm{m}$. It can be seen that the change in the polycrystal response between the two meshes decreases considerably with increasing grain size and $\rho_{i}^{\alpha} / \rho_{\text {ref }}^{\alpha}$ ratio. For $\mathrm{D}=15 \mu \mathrm{m}$, the change in the polycrystal response with mesh refinement is less than $3 \%$, and for $\mathrm{D}=30 \mu \mathrm{m}$, the response becomes mesh insensitive. To further investigate the effect of changing the initial dislocation density in each grain, an appropriate mesh sensitivity measure needs to be defined. For a given grain size and strain level, let that measure be defined in terms of variables for the mesh of interest relative to a mesh-insensitive reference case. Then, a suitable measured may be defined as

$$
\Psi=\frac{\sigma_{C U R}-\sigma_{R E F}}{\eta_{C U R}-\eta_{R E F}}
$$

where $\sigma_{C U R}, \eta_{C U R}$ and $\sigma_{R E F}, \eta_{R E F}$ are the current and reference polycrystal uniaxial stresses and mesh refinement extents (ie. $\eta=\frac{D}{\text { el.size }}$ ), respectively. When $\Psi$ is zero, the polycrystal response is considered to be mesh insensitive. The effect of $\rho_{i}^{\alpha} / \rho_{\text {ref }}^{\alpha}$ on $\Psi$ at $\varepsilon_{33}=0.10$ and $\mathrm{D} \leq 30 \mu \mathrm{m}$ is shown in Fig. 10 . Here, the reference mesh-insensitive case was that corresponding to $\mathrm{D}=30$ $\mu \mathrm{m}$ and $\rho_{i}^{\alpha} / \rho_{\text {ref }}^{\alpha}=625$. It can be seen that smaller values of $\Psi$ are associated with the larger grain sizes. The decrease of $\Psi$ with increasing $\rho_{i}^{\alpha} / \rho_{r e f}^{\alpha}$ ratios is initially sharp and the response becomes approximately mesh insensitive when the $\rho_{i}^{\alpha} / \rho_{\text {ref }}^{\alpha}$ value is raised to 625 . From these results, it is clear that a mesh insensitive polycrystal response can be achieved for a given grain size only for grains with sufficiently high initial dislocation densities (i.e. high values of $\left.\rho_{i}^{\alpha} / \rho_{r e f}^{\alpha}\right)$. Of course, this can only be a solution if justified by available experimental data, as will be discussed next. 


\subsection{Hall-Petch effect on initial yield}

It has been experimentally observed that, for sufficiently fine grains, the initial yield stress of undeformed pure FCC polycrystals, such as $\mathrm{Cu}$ (Carreker and Hibbard, 1953) and Al (Hansen, 1977), are grain-size dependent. This observed Hall-Petch effect at initial yielding cannot be described using the current non-local crystallographic model since no GNDs are accumulated prior to yielding. As discussed by Kubin and Mortensen (2003), this size effect at yield is commonly associated with large number of dislocations at or close to the grain boundary whereas in the work-hardening regime, the effect is governed by the evolution of GNDs.

In this study, to capture the Hall-Petch effect at yield, a unique initial SSD density has been defined for different grain sizes. In order to determine the initial density for each grain size, simulations were carried out on the 8-grain $\mathrm{Cu}$ aggregate for the range of grain sizes taken from the experimental work of Hansen (1979), namely D $=14,33$ and $220 \mu \mathrm{m}$. In all cases, the 10x10x10 FE mesh was used. Suitable values of $\rho_{i}^{\alpha}$ were determined by scaling the $\rho_{i}^{\alpha} / \rho_{\text {ref }}^{\alpha}$ ratio linearly with $D^{-1 / 2}$ (see Fig. 11). From the simulations, the proof and flow stresses at $\varepsilon_{33}=0.002$ and 0.05 are plotted against $D^{-1 / 2}$ in Fig. 11(b) and linearly fitted to obtain the corresponding slopes of 4.34 and 5.12, respectively. The only suitable data from Hansen (1979) that can be used for comparison is at $\varepsilon_{33}=0.05$, where the slope of stress to $D^{-1 / 2}$ was found to be 5.0. Hence, the appropriate $\rho_{i}^{\alpha} / \rho_{\text {ref }}^{\alpha}$ values for $\mathrm{D}=14,33$ and $220 \mu \mathrm{m}$ are chosen to be 127, 77 and 29, respectively. The polycrystal responses predicted for these grain sizes are compared against the data in Fig. 12, where a good agreement can be seen even though only 8 grains have been used to represent an entire polycrystalline aggregate.

For each grain size, additional simulations were also carried out with the strain gradient effects removed (see dashed lines in Fig. 12) so that the hardening behaviour will only be controlled by the evolution of the SSDs. Apart from the largest polycrystal grain size $(\mathrm{D}=220 \mu \mathrm{m})$, where the strain gradient effects are expected to be small in any case, these results revealed that there is a considerable difference between the predicted responses, with and without the evolution of the GNDs. These results also showed that work-hardening contribution from the GNDs is not reduced when the initial SSD population was raised in accordance with the Hall-Petch effect at yield. It is clear to see that the build up of strain gradients, leading to the accumulation of GNDs during deformation, significantly affects the hardening behaviour of the polycrystal.

\section{Conclusions}

A non-local crystallographic model has been relied upon to study the effect of microstructural and deformation-related length scales on the macroscopic 
response of $\mathrm{Cu}$ polycrystalline aggregates. It was found that two distinct phenomena had to be accounted for in order to predict consistently experimentally observed grain size effects in $\mathrm{Cu}$ polycrystals: (i) a Hall-Petch type effect at initial yielding, by assigning higher initial dislocation densities to the smaller grains, and (ii) the generation of geometrically necessary dislocations arising from the slip gradients which develop during deformation.

When the initial yielding of the $\mathrm{Cu}$ polycrystal aggregate was assumed to be independent of grain size, the results were found to be mesh sensitive for grains smaller than $30 \mu \mathrm{m}$ when the initial dislocation densities defined in each grain were relatively low. In such cases, the GNDs generated as a result of the first slip gradients far exceeded the density of SSDs, thus enabling the GNDs to dictate the hardening behaviour of the polycrystal from the onset of deformation. This problem cannot be overcome by further mesh refinement, since even more severe slip gradients, and hence higher GND densities, will develop. Therefore, a mesh insensitive polycrystal response will be obtained for a given grain size only when the width of the slip gradient regions is a small fraction of the grain size, otherwise the grains should contain a sufficiently high amount of dislocations per unit volume before the onset of slip. This study has highlighted some of the strengths and limitations, till now unexplored, of strain gradient formulations which do not rely on higher order fields. A suitable procedure to establish when mesh sensitive results arise has been presented.

\section{Acknowledgements}

We wish to thank Dr. R. Becker for his helpful discussions. This work was performed in part under the auspices of the U.S. Department of Energy by the Univeristy of Calfornia, Lawrence Livermore National Laboratory under Contract W-7405-Eng-48. Financial support from ALSTOM Power (UK) and the Department of Trade and Industry of the United Kingdom are gratefully acknowledged.

\section{References}

ABAQUS V6.2, 2001. Habbitt Karlsson and Sorensen Inc., Providence, RI, USA.

Acharya A., Beaudoin A. J., 2000. Grain-size effect in viscoplastic polycrystals at moderate strains. J. Mech. Phys. Solids 48, 2213-2230.

Acharya A. and Bassani J. L., 2000. Lattice incompatibility and a gradient theory of crystal plasticity. J. Mech. Phys. Solids, 48, 1565-1595.

Arsenlis, A., and Parks, D.M., 2001. Modeling the evolution of crystallographic dislocation density in crystal plasticity, J. Mech. Phys. Solids, 50, 1979-2009. 
Ashby M. F., 1970. The deformation of plastically non-homogeneous materials. Phil. Mag. 21, 399-424.

Bassani, J.L., 2001. Incompatibility and a simple gradient theory of plasticity, J. Mech. Phys. Solids, 49, 1983-1996.

Busso E. P., 1990. Cyclic deformation of monocrystalline nickel aluminide and high temperature coatings. PhD Thesis, MIT.

Busso E. P., Meissonnier F. T. and O’Dowd N. P., 2000. Gradient-dependent deformation of two-phase single crystals. J. Mech. Phys. Solids 48, 23332361.

Busso E. P. and Cheong K. S, 2001. Length scale effects on the macroscopic behaviour of single and polycrystalline FCC crystals. J. Phys. IV 11, Pr(5)161169.

Carreker Jr. R. P. and Hibbard Jr. W. R., 1953. Tensile deformation of highpurity copper as a function of temperature, strain rate, and grain size. Acta Metallurgica 1, 654-663.

Cermelli, P. and Gurtin, M.E., 2001. On the characterization of geometrically necessary dislocations in finite plasticity. J. Mech. Phys. Solids 49, 15391568.

Cheong K. S. and Busso E. P., 2003. Discrete dislocation density modeling of pure single-phase FCC crystals. Submitted for publication.

Dai H. and Parks. D. M., 1997. Geometrically-necessary dislocation density and scale-dependent crystal plasticity. Proceedings of Sixth International Symposium on Plasticity, in: Khan., A. (Ed.), Gordon \& Breach 48.

Essmann U. and Mughrabi H., 1979. Annihilation of dislocations during tensile and cyclic deformation and limits of dislocation densities, Phil. Mag. A 40 (6), 731-756.

Fleck N. A., Muller G. M., Ashby M.F. and Hutchinson J. W., 1994. Strain gradient plasticity : theory and experiment. Acta Metall. 42, 475-487.

Gao H., Huang Y., Nix W. D. and Hutchinson J. W., 1999. Mechanism-based strain gradient plasticity. J. Mech. Phys. Solids 47, 1239-1263.

Gudmundson P., 2004, A unified treatment of strain gradient plasticity. J. Mech. Phys. Solids 52, 1379-1406.

Gurtin, M.E., 2003, On a framework for small deformation visco-plasticity: free energy, microforces, strain gradients. Int. J. Plasticity, 19, 47-90.

Hall E. O., 1951. The deformation and ageing of mild steel. Phys. Soc. of London Proc. 64, 747-753.

Hansen N., 1977. The effect of grain size and strain on the tensile flow stress of aluminium at room temperature. Acta Metallurgica 25, 863-869.

Hansen N., 1979. The effect of grain size and strain on the tensile flow stress of copper at room temperature, in : Proceedings of the 5th International Conference on the Strength of Metals and Alloys 2, 849-854.

Huang Y., Qu S., Hwang K. C., Li M., Gao H., 2004. A conventional theory of mechanism-based strain gradient plasticity, Int. J. Plasticity 20, 753-782.

Honeycombe R. W. K., 1968. The plastic deformation of metals. Edward Arnold Publishers Ltd.

Kocks U. F., Argon A. S. and Ashby M. F., 1975. Thermodynamics and ki- 
netics of slip, in : Progress in Materials Science - Vol. 19, Pergamon Press, Oxford.

Kubin L. P., Mortensen A., 2003. Geometrically necessary dislocations and strain-gradient plasticity : a few critical issues. Scripta Materialia 48, 119125.

Mader S., 1957. Elektronmikroskopische Untersuchung der Gleitlinienbildung auf Kupfereinkristallen. Z. Physik 149, 73-102.

Mecking H. and Kocks U. F., 1981. Kinetics of flow and strain hardening. Acta Metallurgica 29, 1865-1875.

Meissonnier F. T., Busso E. P. and O'Dowd N. P., 2001. Finite-element implementation of a generalised non-local rate-dependent crystallographic formulation for finite strains. Int. J. of Plasticity 17 (4), 601-640.

Nye J. F., 1953. Some geometrical relations in dislocated crystals. Acta. Metallurgica 1, 153-162.

Petch N. J., 1953. The cleavage strength of polycrystals. J. Iron and Steel Inst., 174, 25-28.

Rebstock H., 1957. Kombinierte Zug-und Torsionsverformung von KupbferEinkristallrohen. Z. Metallk. 48, 206-220.

Shu, J.Y., and Fleck, N.A., and Van der Giessen, E., and Needleman, A.,2001, Boundary layers in constrained plastic flow: comparison of nonlocal and discrete dislocation plasticity. J. Mech. Phys. Solids, 49, 1361-1395.

Takeuchi T., 1975. Work hardening of copper single crystals with multiple glide orientations. Trans. Japanese Ins. of Mat. 16, 630-639.

Takeuchi T., 1976. Orientation dependence of work hardening of copper single Crystals near the [001] axis. J. Phys. Soc. Japan 3 (40), 741-746. 


\section{Contact Addresses of Authors}

Dr. Ke-Shen Cheong

Materials Performance Technologies

Industrial Research Limited

69 Gracefield Road, Gracefield

Lower Hutt 6009, New Zealand

Tel : +64-4-931-3092

Email : k.cheong@matperf.com

Dr. Esteban P. Busso

Department of Mechanical Engineering

Imperial College, London

Exhibition Road

London, SW7-2AZ, United Kingdom

Tel : +44-20-7594-7084

Email : e.busso@ic.ac.uk

Dr. Athanasios Arsenlis

Lawrence Livermore National Laboratory

P.O. Box 808, L-371

Livermore, CA 94551-0808, USA

Tel : +1-925-424-2584

Email : arsenlis@llnl.gov 


\section{List of Figures}

1 Procedure to calculate the slip rate gradients at the element integration points (adapted from Busso et al., 2000).

2 Calibrated single crystal model with [100], [111] and [112] oriented copper single crystals. Data taken from Takeuchi (1975).

3 Stereographic projections of randomly assigned grain orientations in copper polycrystals with (a) 8 and (b) 64 grains.

4 Mesh refinement of the 8-grain polycrystal RVE with initial grain of size D.

5 Effect of the grain size D on the overall stress-strain response of the 8-grain polycrystal.

6 Effect of mesh refinement for the 8-grain polycrystal when D is (a) $150 \mu \mathrm{m} \mathrm{(b)} 75 \mu \mathrm{m} \mathrm{(c)} 30 \mu \mathrm{m}$ (d) $15 \mu \mathrm{m}$ (e) $7.5 \mu \mathrm{m}$. Note the increase in mesh sensitivity as $\mathrm{D} \leq 30 \mu \mathrm{m}$.

$7 \quad$ Effect of mesh refinement, for different grain sizes, on the predicted flow stress of the 8-grain polycrystal at $\varepsilon_{33}=0.10$.

8 Predicted polycrystal response for the 8-grain and 64-grain polycrystals, (a) mesh insensitive at $\mathrm{D}=75 \mu \mathrm{m}$ (b) mesh sensitive at $\mathrm{D}=15 \mu \mathrm{m}$.

$9 \quad$ Effect of the initial dislocation density $\left(\rho_{i}^{\alpha}\right)$ on mesh convergence for grain sizes of (a) 7.5, (b) 15 and (c) $30 \mu \mathrm{m},\left(\rho_{\text {ref }}^{\alpha}=16,000\right.$ $\left.\mathrm{mm}^{-2}\right)$.

10 Effect of $\rho_{i}^{\alpha} / \rho_{\text {ref }}^{\alpha}$ on the mesh sensitivity measure $(\Psi)$ for $\mathrm{D}=7.5$, 15 and $30 \mu \mathrm{m}$.

11 (a) Hall-Petch type behaviour at initial yield given by the relation between $\rho_{i}^{\alpha} / \rho_{\text {ref }}^{\alpha}$ and $D^{-1 / 2}$, and (b) the corresponding predicted effect of $D^{-1 / 2}$ on the flow stress, $\sigma_{33}$, at two different strain levels, $\varepsilon_{33}=0.002$ and 0.05 .

12 Comparison between the predicted polycrystal response of the 8-grain polycrystals with the experimental data of Hansen (1979) for $\mathrm{D}=14,33$ and $220 \mu \mathrm{m}$. Dashed lines show the respective predictions when strain gradient effects are removed. 


\section{List of Tables}

$1 \quad$ Single crystal model parameters 

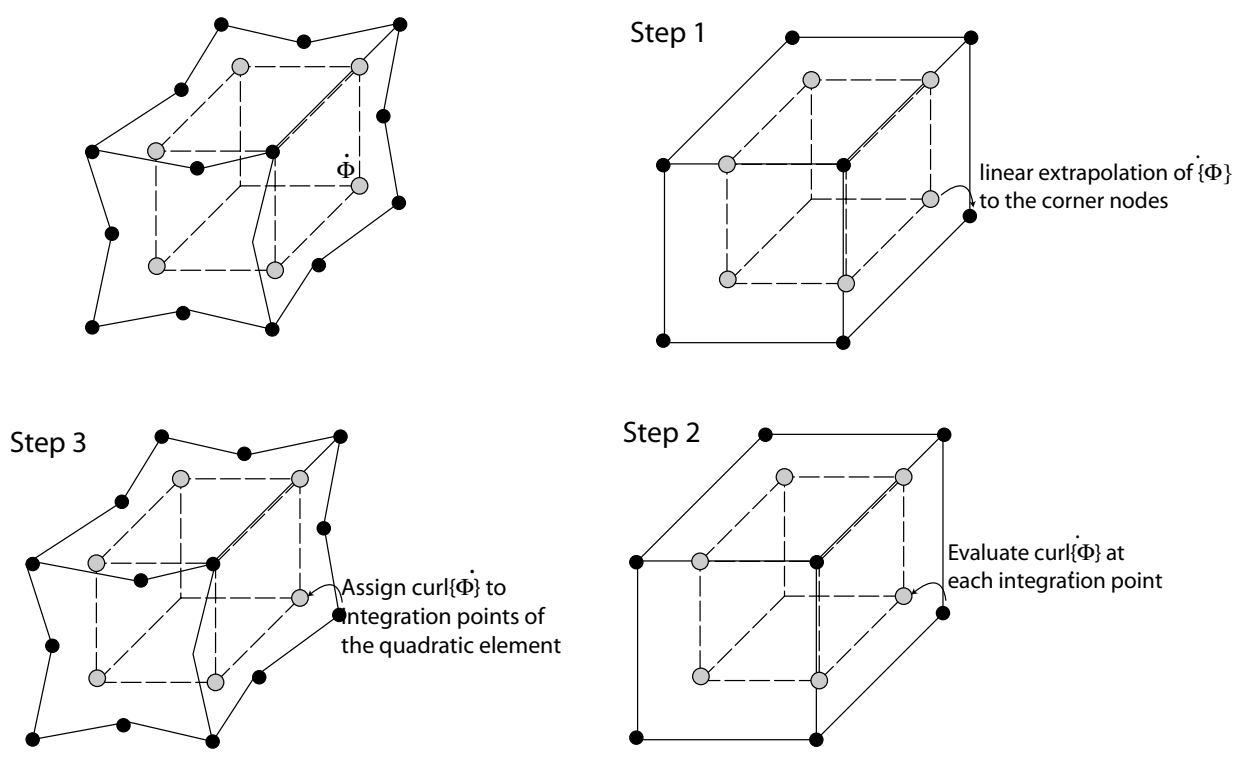

Fig. 1. Procedure to calculate the slip rate gradients at the element integration points (adapted from Busso et al., 2000).

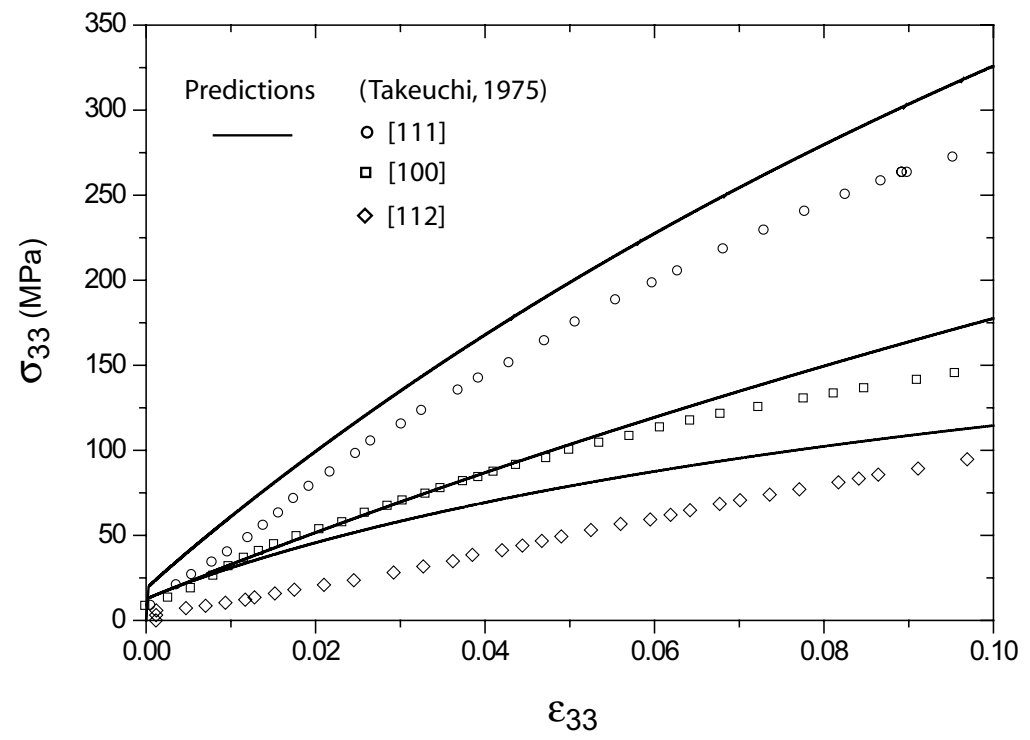

Fig. 2. Calibrated single crystal model with [100], [111] and [112] oriented copper single crystals. Data taken from Takeuchi (1975). 


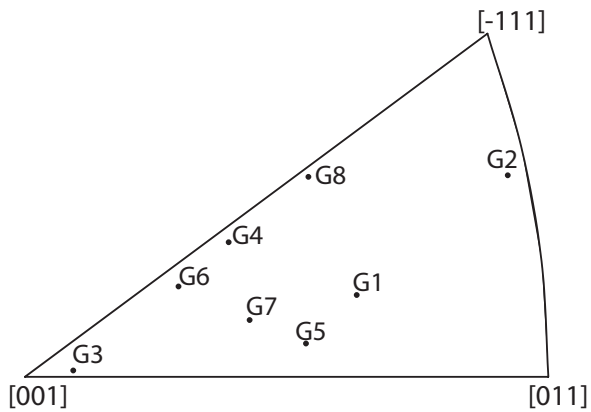

( a )

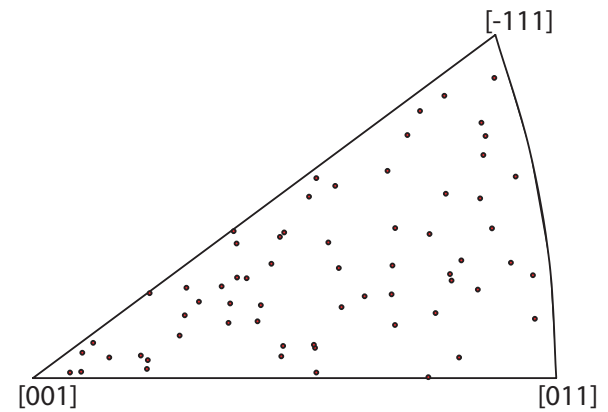

( b )

Fig. 3. Stereographic projections of randomly assigned grain orientations in copper polycrystals with (a) 8 and (b) 64 grains.
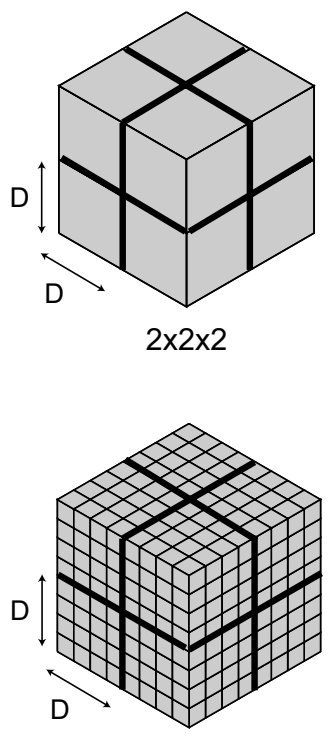

$8 \times 8 \times 8$
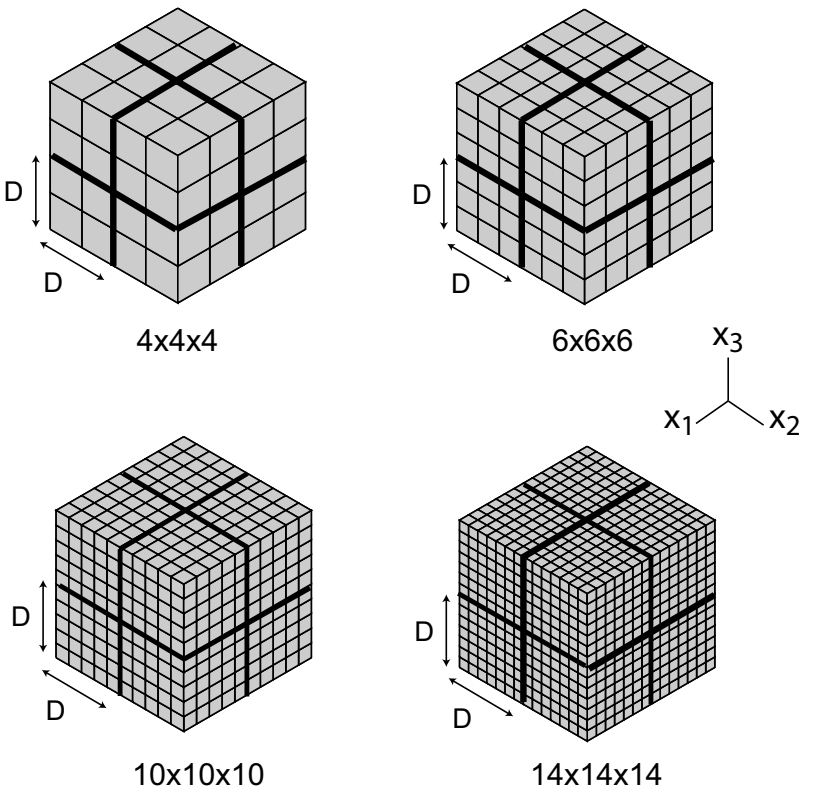

Fig. 4. Mesh refinement of the 8-grain polycrystal RVE with initial grain of size D. 


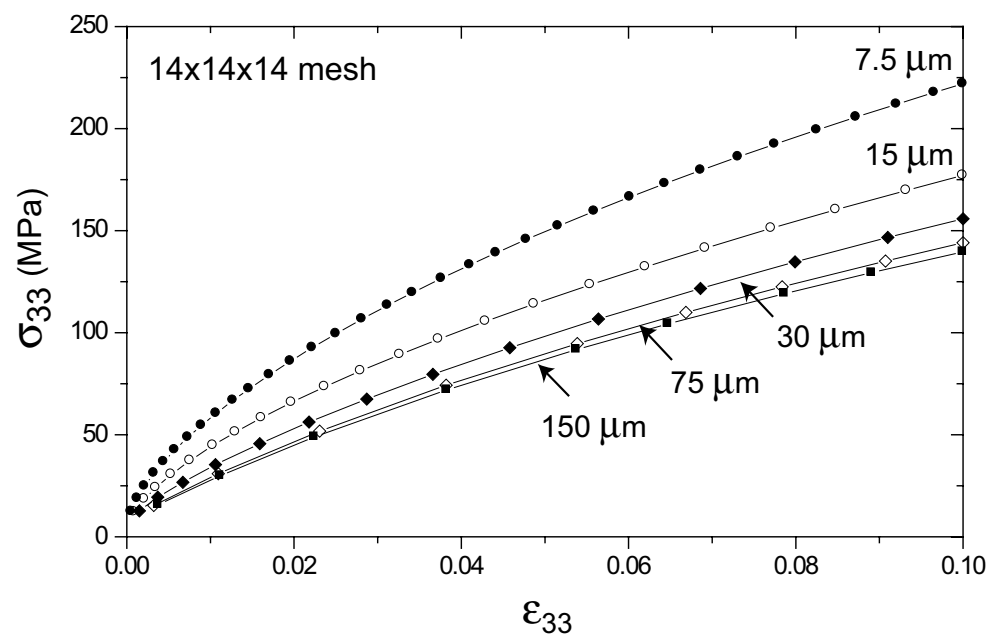

Fig. 5. Effect of the grain size D on the overall stress-strain response of the 8-grain polycrystal. 


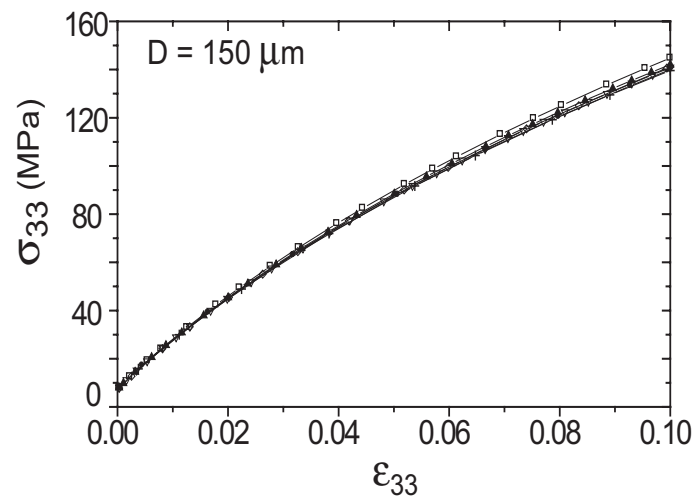

(a)

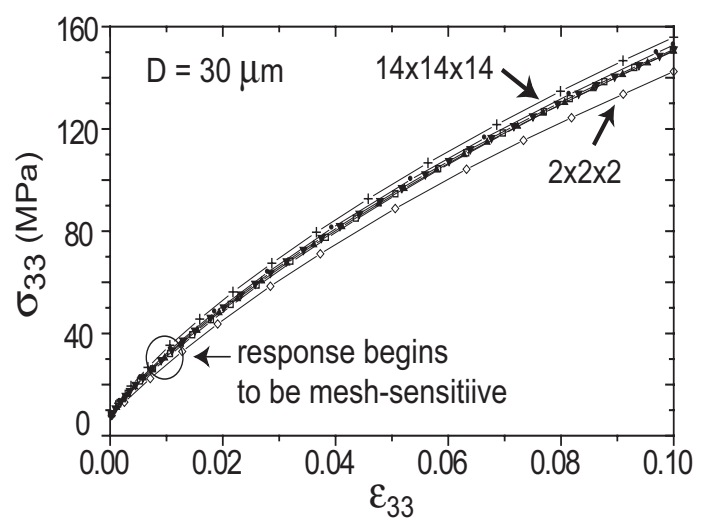

(c)

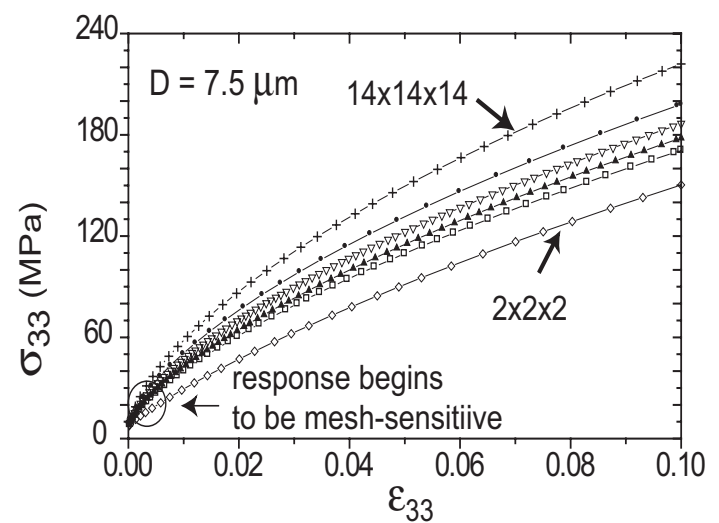

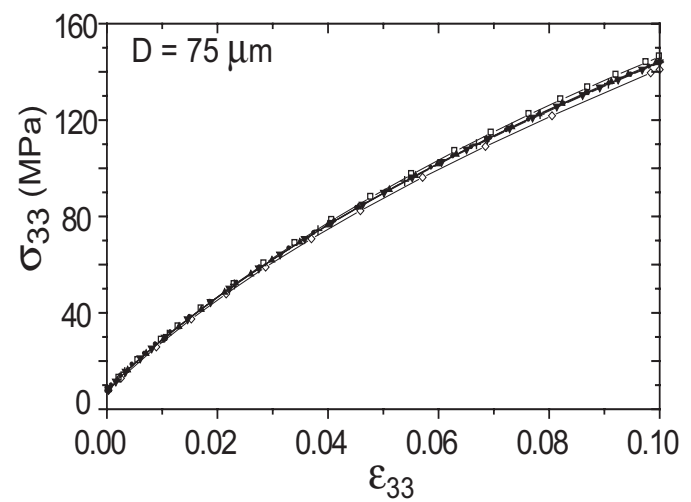

(b)

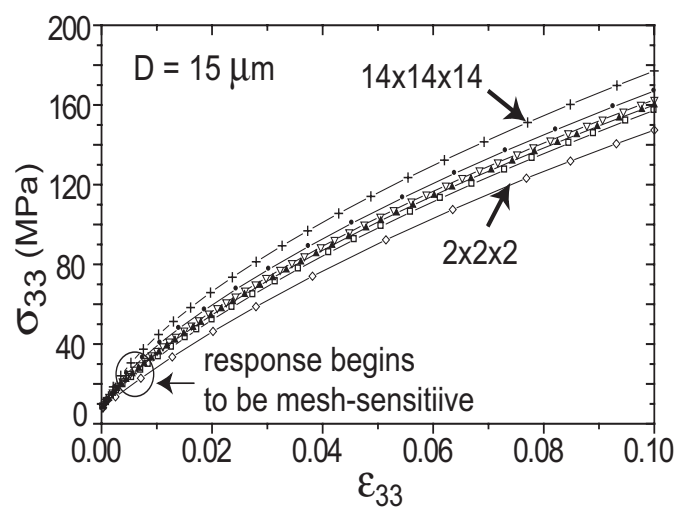

(d)

(e)

Fig. 6. Effect of mesh refinement for the 8-grain polycrystal when D is (a) $150 \mu \mathrm{m}$ (b) $75 \mu \mathrm{m}$ (c) $30 \mu \mathrm{m}$ (d) $15 \mu \mathrm{m}$ (e) $7.5 \mu \mathrm{m}$. Note the increase in mesh sensitivity as D $\leq 30 \mu \mathrm{m}$. 


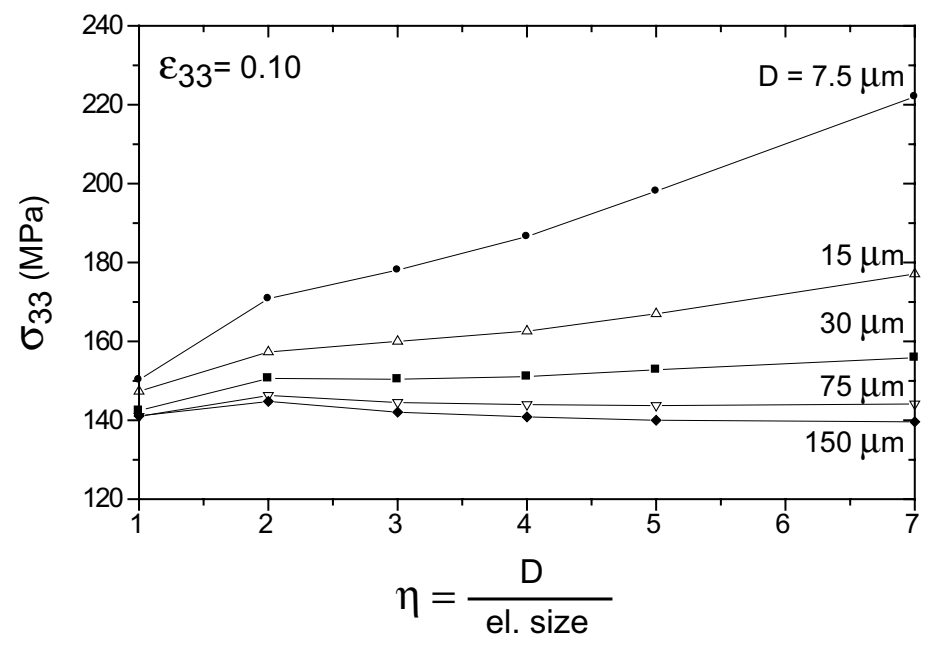

Fig. 7. Effect of mesh refinement, for different grain sizes, on the predicted flow stress of the 8-grain polycrystal at $\varepsilon_{33}=0.10$.

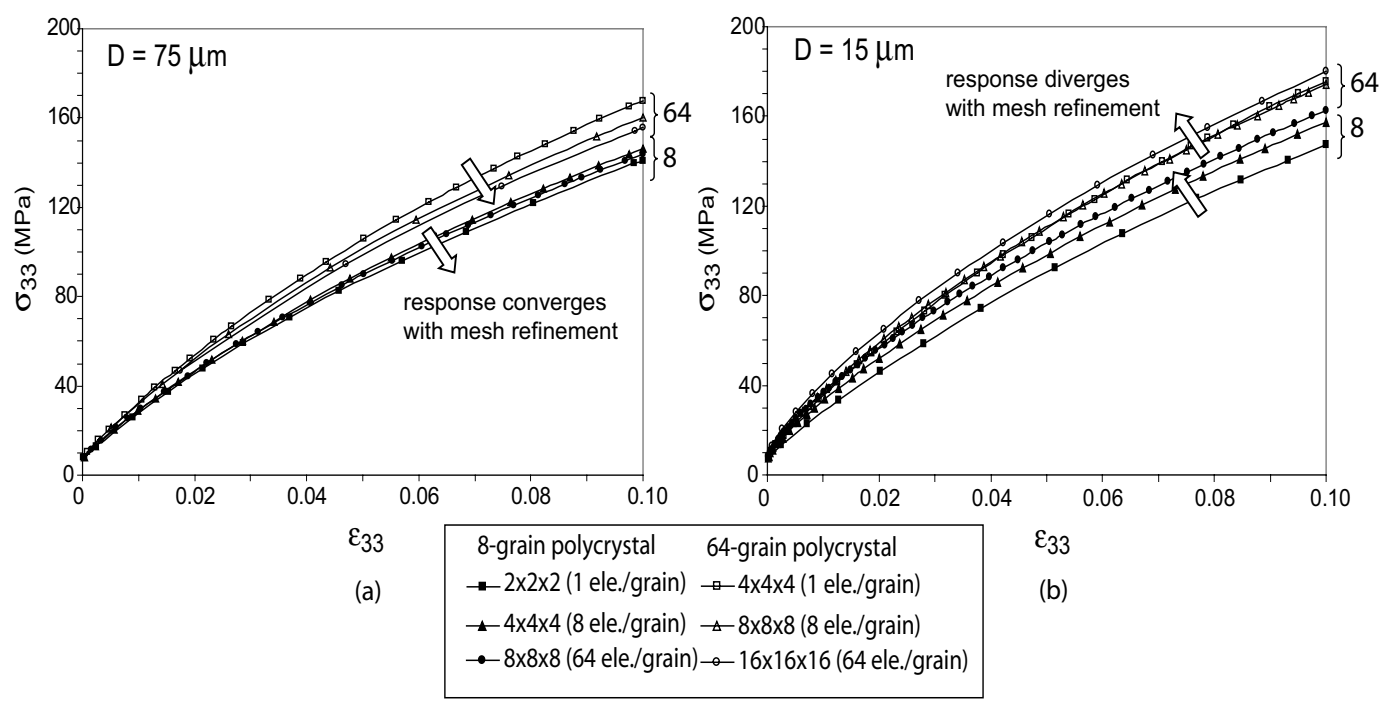

Fig. 8. Predicted polycrystal response for the 8-grain and 64-grain polycrystals, (a) mesh insensitive at $\mathrm{D}=75 \mu \mathrm{m}$ (b) mesh sensitive at $\mathrm{D}=15 \mu \mathrm{m}$. 


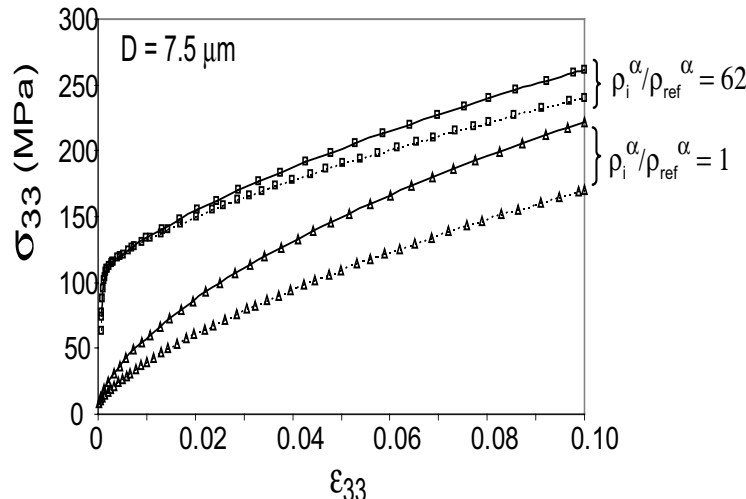

(a)

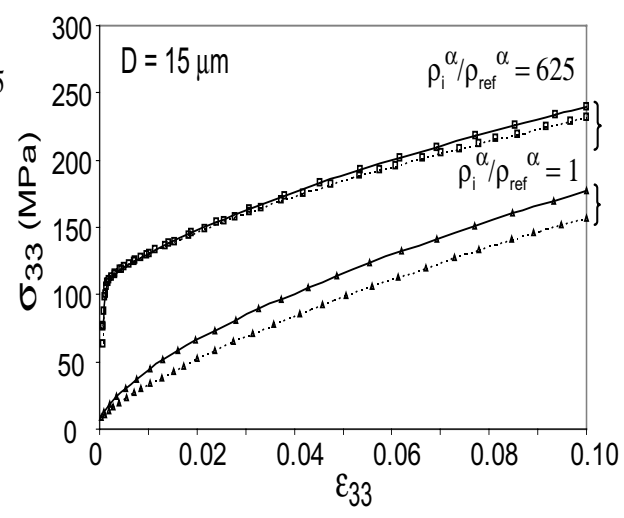

(b)
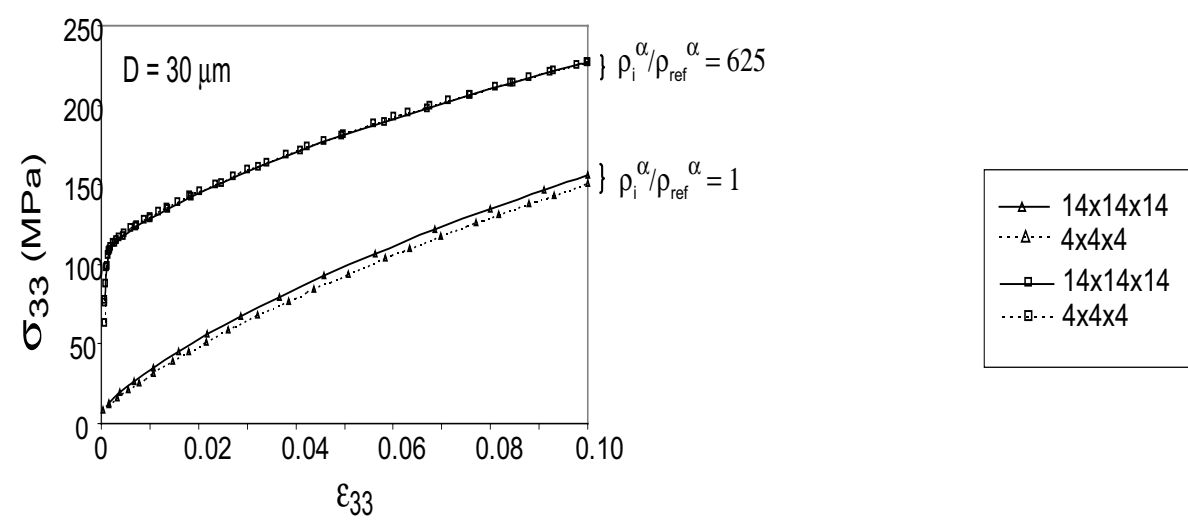

(c)

Fig. 9. Effect of the initial dislocation density $\left(\rho_{i}^{\alpha}\right)$ on mesh convergence for grain sizes of (a) 7.5, (b) 15 and (c) $30 \mu \mathrm{m},\left(\rho_{\text {ref }}^{\alpha}=16,000 \mathrm{~mm}^{-2}\right)$. 


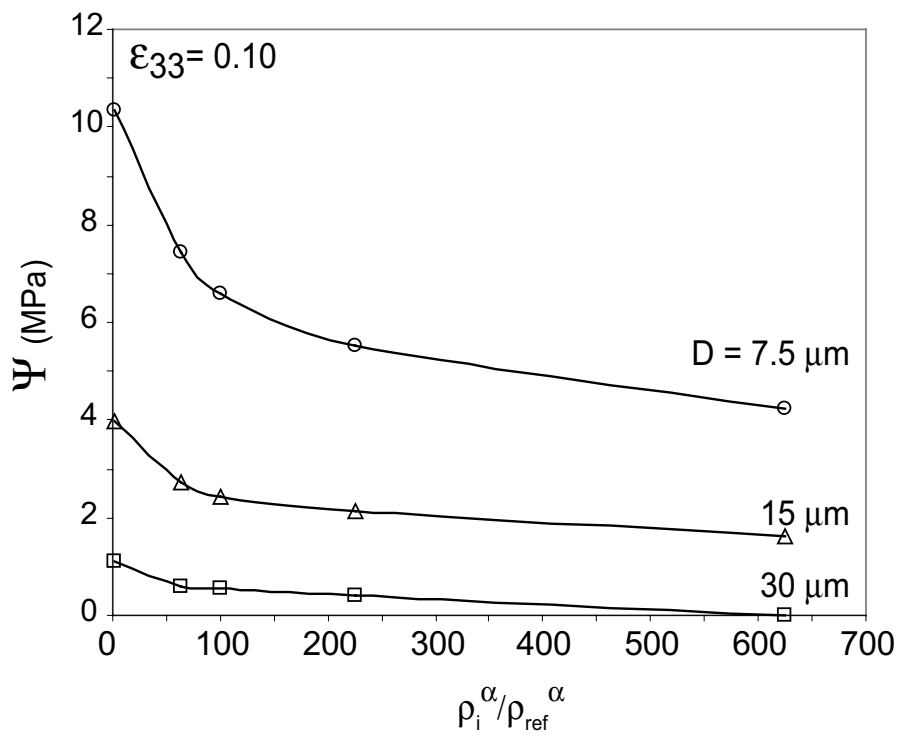

Fig. 10. Effect of $\rho_{i}^{\alpha} / \rho_{\text {ref }}^{\alpha}$ on the mesh sensitivity measure $(\Psi)$ for $\mathrm{D}=7.5,15$ and $30 \mu \mathrm{m}$.

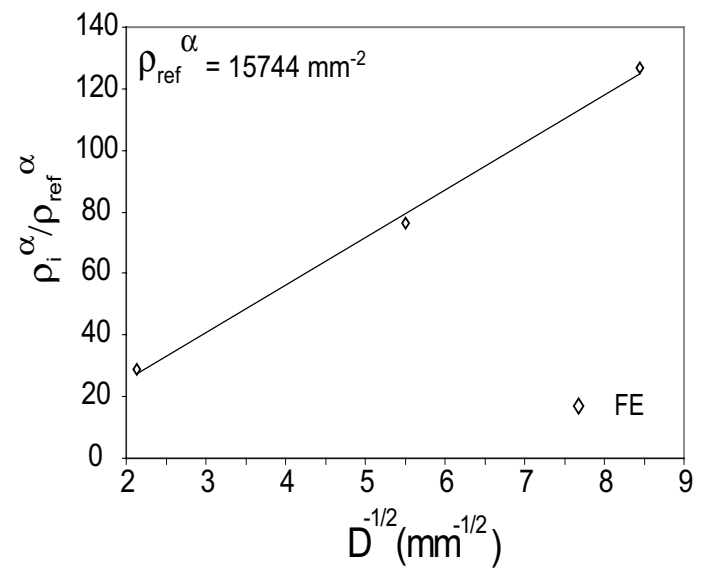

(a)

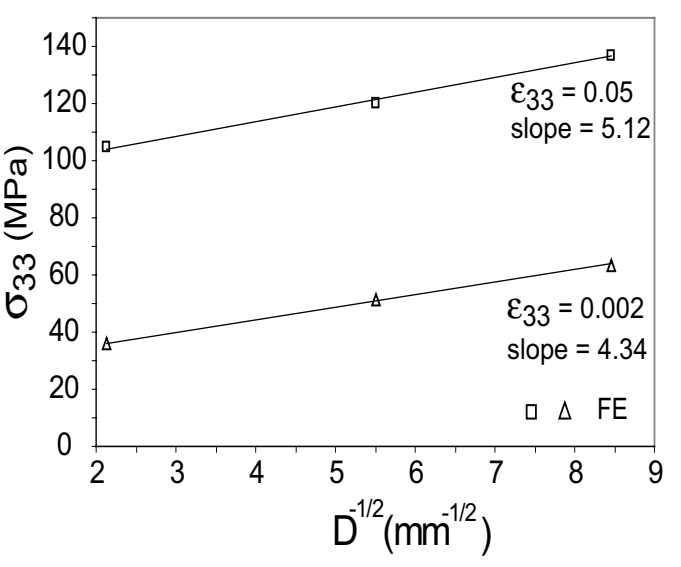

(b)

Fig. 11. (a) Hall-Petch type behaviour at initial yield given by the relation between $\rho_{i}^{\alpha} / \rho_{\text {ref }}^{\alpha}$ and $D^{-1 / 2}$, and (b) the corresponding predicted effect of $D^{-1 / 2}$ on the flow stress, $\sigma_{33}$, at two different strain levels, $\varepsilon_{33}=0.002$ and 0.05 . 


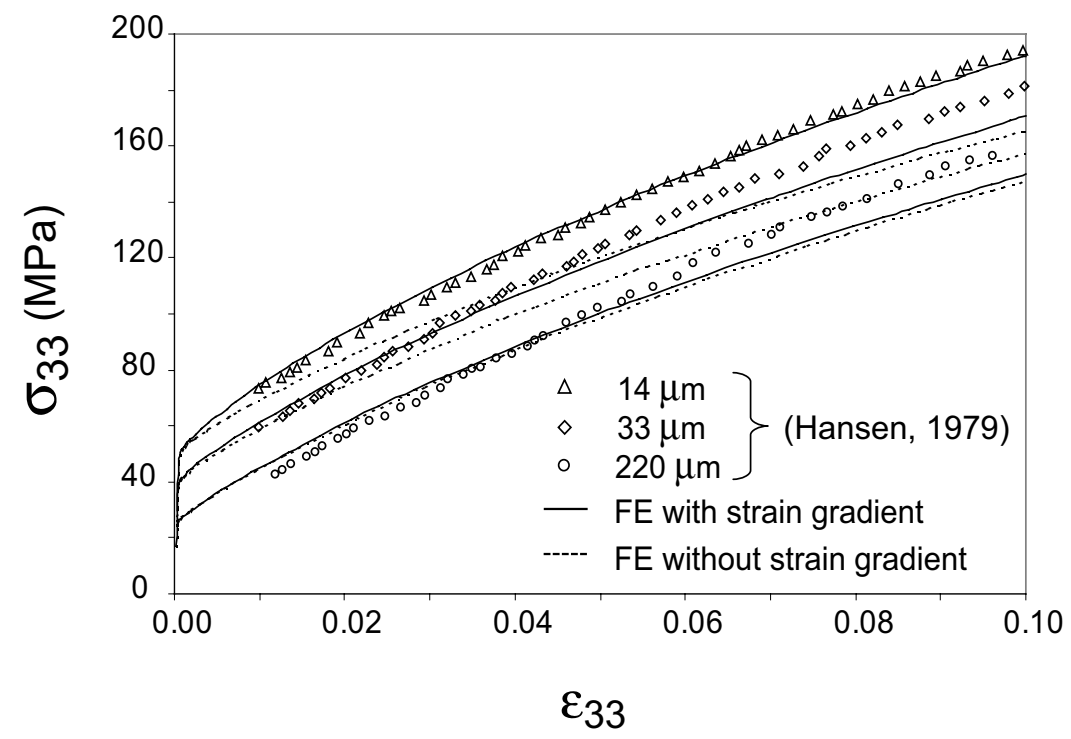

Fig. 12. Comparison between the predicted polycrystal response of the 8-grain polycrystals with the experimental data of Hansen (1979) for D $=14,33$ and $220 \mu \mathrm{m}$. Dashed lines show the respective predictions when strain gradient effects are removed. 
Table 1

Single crystal model parameters

\begin{tabular}{|c|c|c|}
\hline Elastic Constants (GPa) & Flow Rule (Eq.6) & Hardening Laws (Eqs. 9,10) \\
\hline \multicolumn{3}{|c|}{ Material parameters obtained from other than stress-strain data } \\
\hline$C_{11}=166.1$ & & \multirow{8}{*}{$\begin{array}{c}K_{s w}=2 K_{e} \\
b=2.57 \times 10^{-7} \mathrm{~mm} \\
\rho_{S_{e}}^{\alpha}=\rho_{S_{s w}}^{\alpha}=8000 \mathrm{~mm}^{-2} \\
d_{e}=1.0 \mathrm{~nm} ; d_{s w}=5.0 \mathrm{~nm} \\
\omega_{S 1}=1.5 ; \omega_{S 2}=1.2 \\
\omega_{G 1}=0.0 ; \omega_{G 2}=0.0 \\
C_{e}=C_{s w}=0.5 \\
\lambda=0.3\end{array}$} \\
\hline$C_{12}=111.9$ & & \\
\hline$C_{44}=75.6$ & & \\
\hline$\mu_{0}=49.0$ & & \\
\hline \multirow[t]{4}{*}{$\mu=45.0$} & & \\
\hline & & \\
\hline & & \\
\hline & & \\
\hline \multicolumn{3}{|c|}{ Material parameters chosen to fit the stress-strain data } \\
\hline & $F_{0}=2.5 \times 10^{-19} \mathrm{~J}$ & $K_{e}=14.1 \times 10^{-3}$ \\
\hline & $\hat{\tau}_{0}=20.0 \mathrm{MPa}$ & \\
\hline & $\dot{\gamma}_{0}=10^{6} s^{-1}$ & \\
\hline & $p=0.20 ; q=1.20$ & \\
\hline
\end{tabular}

